Nikola Stilinović

Milan Marković

Nikola Mišković

Zoran Vukić

Antonio Vasilijević

http://dx.doi.org/10.21278/brod67305

\title{
MECHANICAL DESIGN OF AN AUTONOMOUS MARINE ROBOTIC SYSTEM FOR INTERACTION WITH DIVERS
}

\author{
UDC 629.5/.01/.051.5:629.563.84
}

Original scientific paper

\section{Summary}

SCUBA diving, professional or recreational, remains one of the most hazardous activities known by man, mostly due to the fact that the human survival in the underwater environment requires use of technical equipment such as breathing regulators. Loss of breathing gas supply, burst eardrum, decompression sickness and nitrogen narcosis are just a few problems which can occur during an ordinary dive and result in injuries, long-term illnesses or even death. Most common way to reduce the risk of diving is to dive in pairs, thus allowing divers to cooperate with each other and react when uncommon situation occurs. Having the ability to react before an unwanted situation happens would improve diver safety.

This paper describes an autonomous marine robotic system that replaces a human dive buddy. Such a robotic system, developed within an FP7 project "CADDY - Cognitive Autonomous Diving Buddy" provides a symbiotic link between robots and human divers in the underwater. The proposed concept consists of a diver, an autonomous underwater vehicle (AUV) Buddy and an autonomous surface vehicle (ASV) PlaDyPos, acting within a cooperative network linked via an acoustic communication channel. This is a first time that an underwater human-robot system of such a scale has ever been developed. In this paper, focus is put on mechanical characteristics of the robotic vehicles.

Key words: diver; underwater robot; navigation; communication; interaction; AUV; ASV

\section{Introduction}

The community of SCUBA divers is increasing each year and some estimates say that there are even as many as seven millions active divers with the number increasing annually [1]. Both recreational (those involved in SCUBA diving for pleasure) and professional (technical divers, search and rescue divers, scientific divers, etc.) divers are constantly jeopardized mostly due to the fact that the environment in which they operate is not suitable for humans. In order to maintain life support, they rely on technical devices (such as breathing 
regulators) which may be prone to malfunctioning. In addition to that, the underwater habitat itself introduced conditions such as nitrogen narcosis that can be fatal for humans. In order to minimize these risks, divers usually pair up. However, the risk still remains. Further on, many challenges involved with diving are related to reduced navigation and communication capabilities underwater due to the absence of global positioning and electromagnetic communication signals. These are commonly replaced by using acoustic signals that are characterized with low bandwidth [2].

In the world of rapid robotics development, it is natural to investigate the possibilities of using these highly sophisticated and advanced technologies for the purpose of minimizing the risk of diving and alleviating the diver during underwater operations.

\subsection{Background}

With the appearance of remotely operated underwater vehicles (ROVs), cooperation between the divers and robots started. ROV operators from the surface would remotely control ROVs to monitor diver activities in order to alleviate communication and ensure quick responsiveness in case of hazards. This cooperation was on a basic level, since almost no autonomy was present in the underwater robots. Only with the uptake of autonomous underwater vehicles (AUVs) did the real human-robot cooperation in the underwater environment start. Deprived of a tether that would connect them to the surface station, AUVs must contain enough autonomy to accomplish a mission, whether it is an underwater survey [3] [4] manipulation [5] [6], or cooperation with divers.

There is only a few mentions of using autonomous marine vehicles in diver-robot interaction. One of the first attempts to aid diver guidance and navigation was performed by Instituto Superior Tecnico who used a fleet of three autonomous marine vehicles to determine diver position by underwater triangulation, and guide the diver through the underwater environment [7]. This work also investigated methods of interacting with a diver in a form of issuing directional commands through LEDs positioned inside the diver goggles.

The group from McGill University investigated vision as the interaction modality for easy programming of underwater robots [8] [9]. The scheme was tested on an amphibious AUV called aqua, which was also used to track diver's motion using visual markers. Substantial work in the area of human-robot interaction in the underwater environment has also been made at Georgia Institute of Technology [10] [11].

\subsection{The concept of Cognitive Autonomous Diving Buddy}

"CADDY - Cognitive Autonomous Diving Buddy" [12] is an FP7 ICT project that envisions a formation of two robots (surface and underwater) that interact with the diver, as shown in Figure 1. While the autonomous surface vehicle (ASV) has the role of the diver's "private satellite", the autonomous underwater vehicle (AUV) plays the role of buddy "observer" that monitors the diver, buddy "guide" that leads the diver through the underwater environment, and buddy "slave" that assists the diver during underwater operations. The ASV provides a precise GPS position for both the diver and the AUV, thus serving as a navigation aid for the diver and the AUV. It is also used as a diver buoy with the diver down flag since it is dynamically positioned to be above the diver. Being a part of this formation, the diver interacts with the companion underwater vehicle that manoeuvres in the vicinity of the diver and reacts accordingly to diver actions. The ASV communicates with the diver and the AUV via acoustic communication and shares the acquired data with the command centre. 
The diver is equipped with the acoustic modem which allows diver tracking and communication. The diver tablet, located in a custom made underwater housing and stylus, gives the diver the possibility to exchange messages with the command centre and, for the first time, to see its position on the map underwater. Full tablet functionality is preserved with the underwater housing and stylus which enables taking photos or videos, writing notes or playing games, reading a book and watching videos during the ascent.

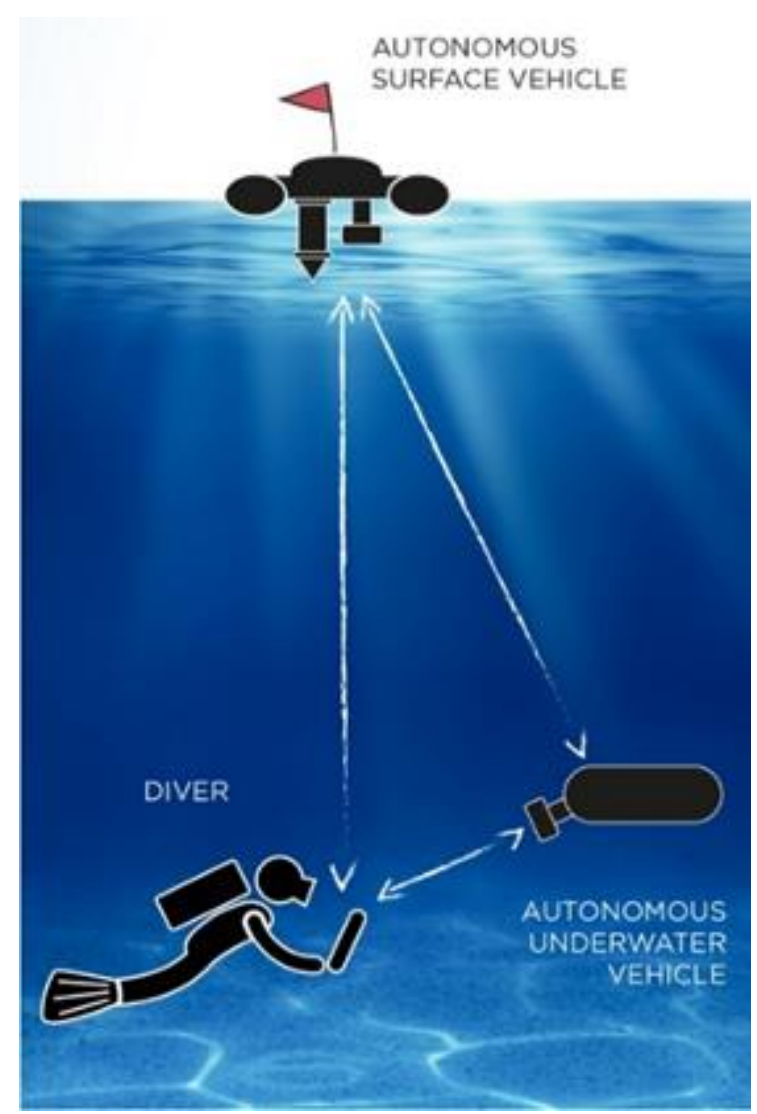

Figure 1 The CADDY concept

In the CADDY project, the primary role of an autonomous surface vehicle is assigned to MedusaS, semi-submersible autonomous marine vehicle developed at the Laboratory of Robotics and Systems in Engineering and Science (LARSyS)/ISR of the Instituto Superior Técnico of Lisbon (IST), Portugal, while the backup vehicle is PlaDyPos ASV, developed at Laboratory for Underwater Systems and Technologies (LABUST), Faculty of Electrical Engineering and Computing (FER) University of Zagreb (UNIZG). Interested reader can refer to [13] to find more information about MedusaS vehicle. The primary role of the autonomous underwater vehicle is taken by a unique vehicle Buddy AUV, also developed at LABUST. The backup AUV called e-URoPe is designed by Consiglio Nazionale delle Ricerche (CNR), a partner in the project.

\subsection{Focus of the paper}

This article focuses on the description of the robotic vehicles comprising CADDY architecture that were developed at LABUST UNIZG-FER, i.e. PlaDyPos ASV and Buddy AUV. The design and main characteristics of both autonomous vehicles is described. Electric schemes are provided with explained communication and wiring inside the vehicles. 3D model of the AUV is shown with all essential components explained.

\section{The autonomous surface vehicle - PlaDyPos}

The initial design of PlaDyPos ASV was a result of s student project with the main goal to build a small, one man portable overactuated platform to perform dynamic positioning algorithms, hence its name PlaDyPos (platform for dynamic positioning). During the four years from its first assembly and after couple of iterations, PlaDyPos evolved into multipurpose vehicle (Figure 2) with a great number of sensors, capable of executing numerous guidance and control tasks in sea state 3 on the Douglas Sea Scale. Dimensions of the vehicle are $707 \times 707 \times 450 \mathrm{~mm}$ and the mass is about $35 \mathrm{~kg}$. This vehicle in CADDY project provides navigation and communication link between a diver, AUV and ground station. The hull of the ASV is made of glass reinforced polyester. It is actuated by four thrusters positioned in such a way that they form an X-shaped configuration (Figure 3) which allows movement in every direction while maintaining arbitrary desired heading. The ASV 


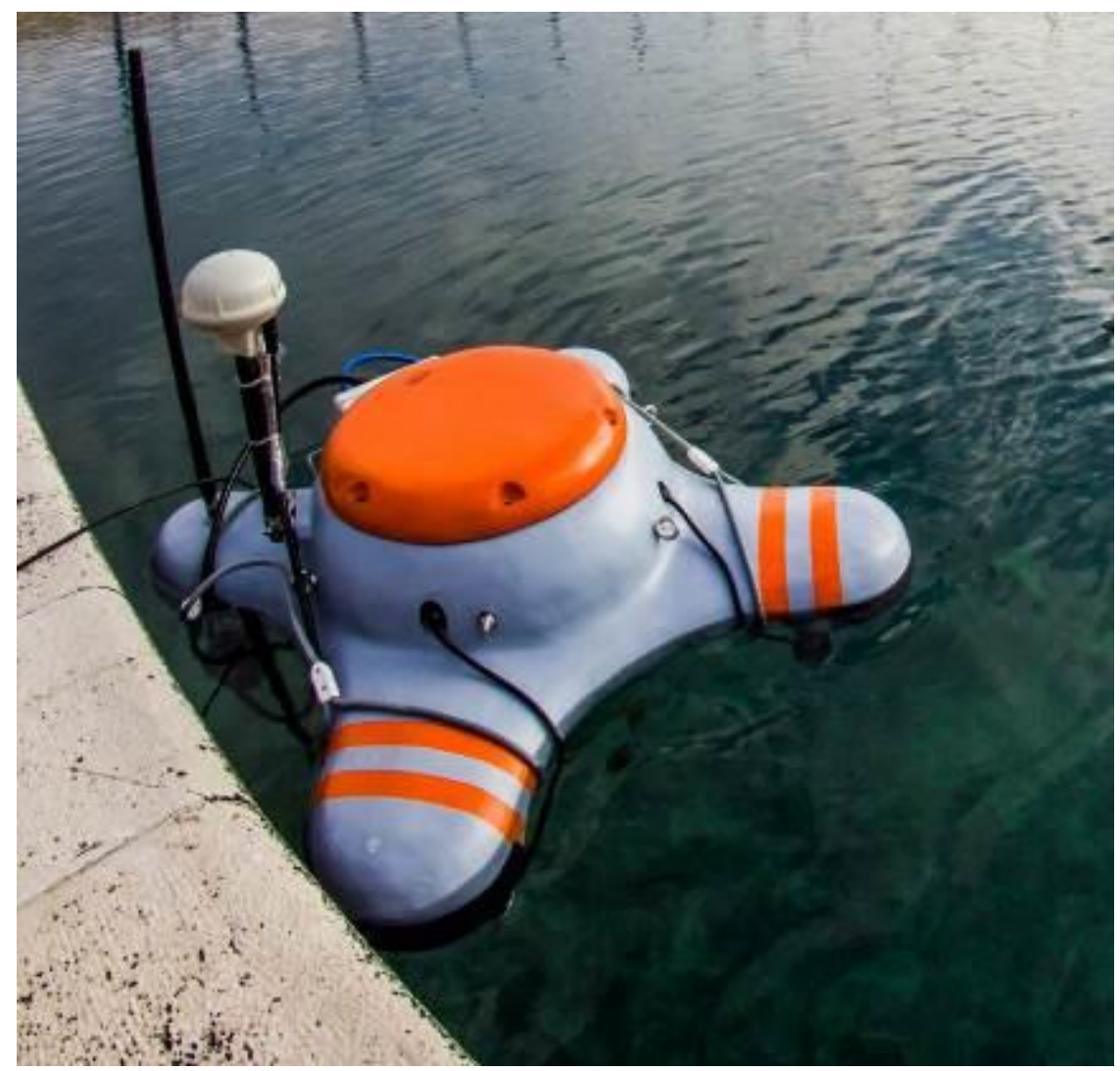

Figure 2 The autonomous surface vehicle PlaDyPos has Inertial Measurement Unit (IMU) and Real Time Kinematic Global Positioning System (RTK GPS) for navigation on the water surface, Ultra-Short Base Line (USBL) for acoustic localisation and communication with underwater agents e.g. divers $[14] \quad$ or underwater vehicles [15] and down looking high definition (HD) camera for diver tracking [16] or shallow water mosaicking [17]. RTK GPS uses two GPS antennas; one is on the ASV (rover), while the other is ground-based station (base). The latter GPS antenna is a reference station positioned at an accurately-known fixed location. If exact position is unknown, the base station averages the current antenna position over a period of time to obtain the best of location data accuracy. Instead of using the information content of the signal, RTK GPS uses measurements of the phase of the signal's carrier wave and provides real-time corrections. With this system it's possible to have centimetre-level accuracy, or better.

Apart from the sensors used in CADDY project, the vehicle is capable of working with variety of different sensors (Doppler Velocity Log (DVL), sonar, stereo camera, etc.) illustrated in Figure 4.

Inside the hull of the ASV is electronic system powered with $12 \mathrm{~V} 500$ Wh AGM gel battery. Intel ${ }^{\circledR}$ NUC i5 mini $\mathrm{PC}$ is responsible for gathering data from the sensors and for navigation, guidance and control (NGC) of the vehicle. The communication inside the vehicle is based on Ethernet: every electronic part which is not directly connected to main computer is connected by Ethernet via Ethernet switch. The ASV is accessible from the ground station through Ubiquiti Bullet ${ }^{\mathrm{TM}}$ M2 Wi-Fi antenna. Each of the ASV devices, sensors or thrusters can be individually and wirelessly turned on/off via Ethernet relay board. BlueRobotics T200 thrusters are cavity-free thrusters with brushless motor. Motors are sealed and protected with an epoxy coating so water can

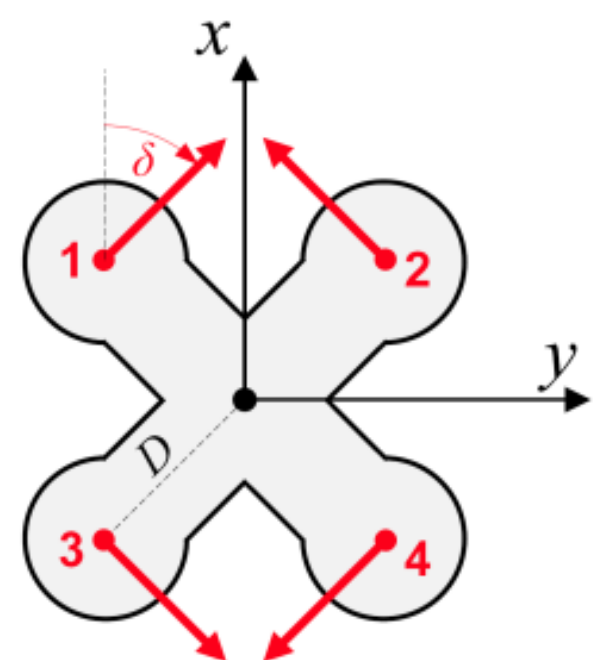

Figure 3 Thruster orientation with $\delta=45^{\circ}$ 
Mechanical Design of an Autonomous Marine Robotic System for Interaction with Divers
Nikola Stilinović, Milan Marković

Nikola Mišković, Zoran Vukić, Antonio Vasilijević

flow freely through all parts of the motor, making it water-cooled motor being able to withstand extreme pressures. For being able to setup allocation matrix of the thrusters, it was necessary to measure forces which each thruster gives. Figure 5 shows the graph of the thruster force against the PWM input. The thrusters are fixed on $45^{\circ}$ angle from $\mathrm{X}$ and $\mathrm{Y}$ axis of the ASV. This configuration represents a trade-off solution providing equal performance in $\mathrm{X}$ and $\mathrm{Y}$ direction, important for vehicle designed for precision dynamic positioning but paid by lose of efficiency. The overall loss is about $30 \%\left(\cos \left(45^{\circ}\right)=0.7071\right)$ per motor for surge and sway motion.

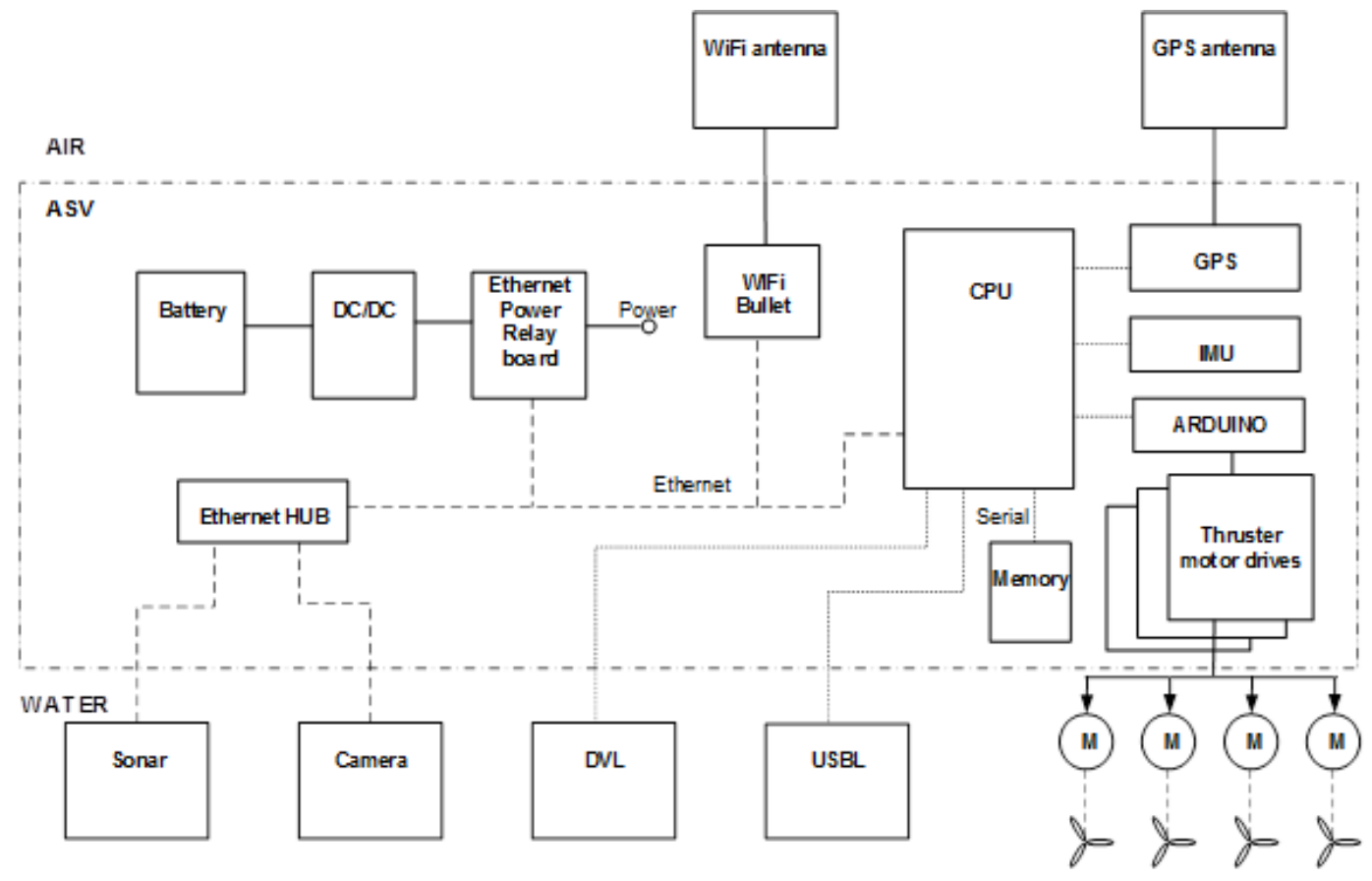

Figure 4 Electrical scheme of the ASV with all possible sensor configurations

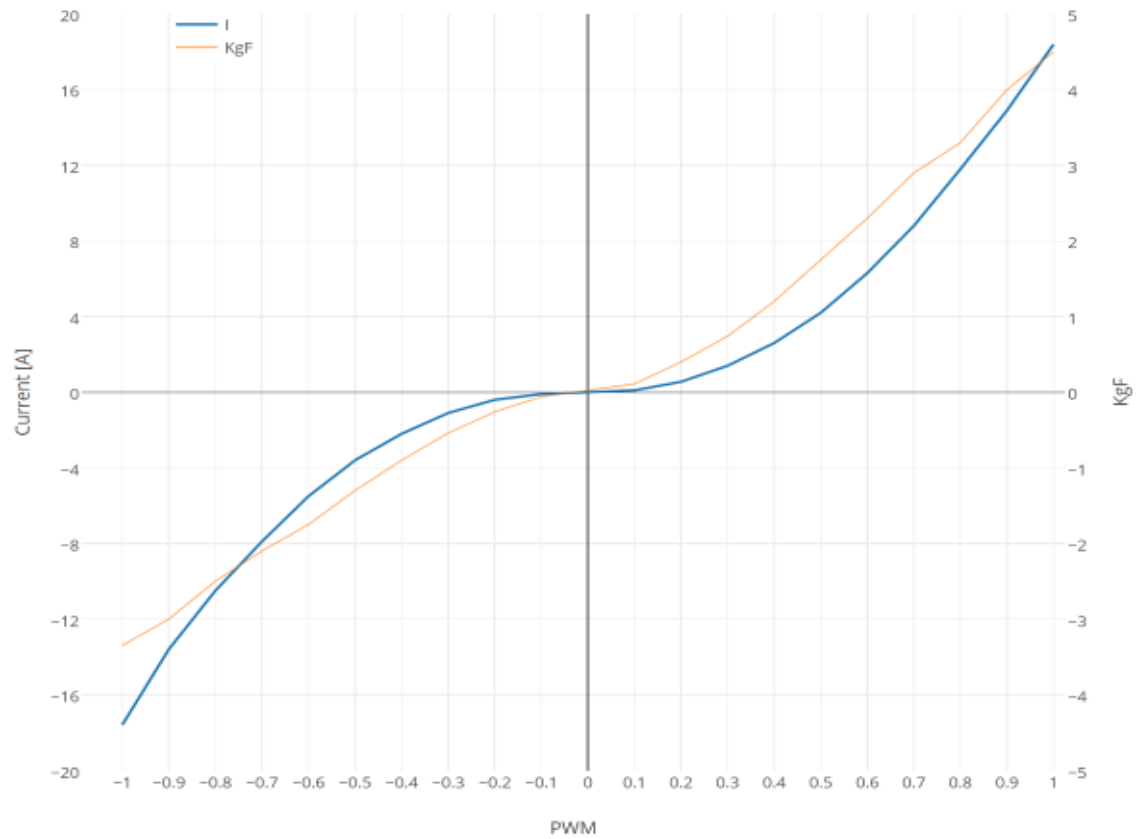

Figure 5 Thrust mapping of T200 thrusters 


\section{The autonomous underwater vehicle - Buddy}

The main task of AUV is to support diver operations. The AUV has high manoeuvring capabilities (full actuation) due to four horizontal and two vertical thrusters and is capable of dealing effectively with underwater environment. Integrated on the AUV are proprioceptive sensor devices needed to develop a precise navigation module, i.e. IMU, DVL and USBL based positioning system. To be able to recognize postures and gestures of the diver, a set of exteroceptive sensors (stereo video system and sonar) are integrated. An acoustic based communication link, as already mentioned in section 2 is integrated to ensure the communications between the underwater and surface segment and also with the diver.

\subsection{Hardware construction of Buddy AUV}

The frame of the autonomous underwater vehicle is made of an engineered plastic (Polyacetal, POM-C) (Figure 6). All pieces were cut from $9 \mathrm{~mm}$ thick sheet of plastic and screwed together with stainless steel nuts and bolts. Hinges, lock nuts, flanges and mounts are made of Aluminium alloy. Overall dimensions of the vehicle are 1220x700x750 mm and the weight is about $70 \mathrm{~kg}$. Three waterproof Aluminium canisters contain electronics and the battery which will be explained in 4.1 .
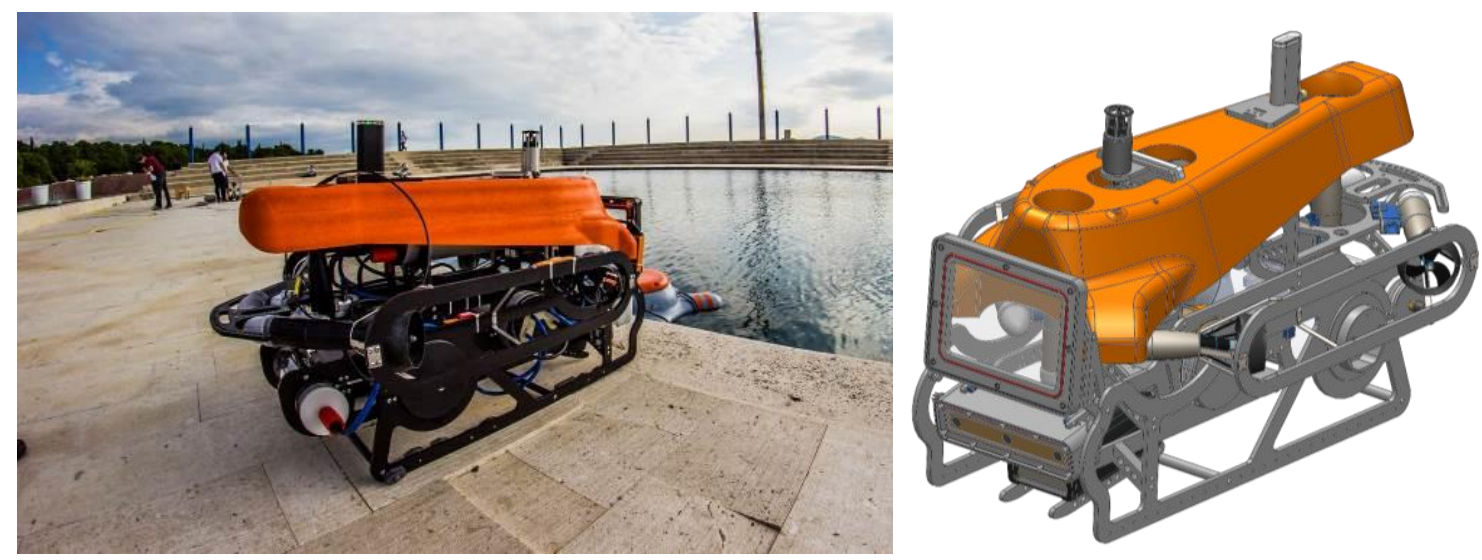

Figure 6 The autonomous underwater robot Buddy during trials and 3D representation

The vehicle carries a large variety of sensors. Navigation sensor suite consist of:

- USBL - AUV and ASV are equipped with BluePrint Subsea X150 USBL beacons [18] while diver carries BluePrint Subsea X110 modem beacon. The system provides position of the agents relative to each other. The absolute coordinates of the Buddy and diver are obtained by merging their relative positions with absolute, GPS position of the ASV [19]. The same unit is used for communication between the agents. USBL unit is shown in exploded view of Buddy AUV in Figure 7.

- IMU - Microstrain's 3DM-GX3®-25 9 axis IMU gives accelerometer, magnetometer and gyro measurements used to estimate attitude and assists the Kalman filter with movement data. As shown in Figure 8 it is located inside the Master canister.

- DVL - Gives altitude as well as velocity over ground in $\mathrm{X}, \mathrm{Y}$ and $\mathrm{Z}$ direction (Figure 7). DVL used in AUV is LinkQuest NavQuest 600 Micro.

- GPS - u-blox Lea series GPS provides absolute position and it is used for navigation on the surface. 
Mechanical Design of an Autonomous Marine Robotic System for Interaction with Divers
Nikola Stilinović, Milan Marković

Nikola Mišković, Zoran Vukić, Antonio Vasilijević

Sensors for remote sensing are (Figure 7):

- Multibeam sonar - High frequency (3.0 and 1.8 MHz) Sound Metrics ARIS Explorer 3000 sonar gives unprecedented high definition imagery of the diver, as shown in Figure 9, from $0.7 \mathrm{~m}$ to 5 or to $15 \mathrm{~m}$, depending on frequency used.

- Stereo camera - Point Grey Bumblebee XB3 3-sensor multi-baseline monochrome camera is used for detecting divers pose when water visibility is high. Figure 10 shows the diver communicating with sign language in 3D image from stereo camera. Images from 3 cameras are fused together and presented in different colours.

- Low-light camera - Bosch FLEXIDOME IP starlight 7000 VR colour camera with $0.017 \mathrm{~lx}, \mathrm{HD}$ resolution and with $180^{\circ}$ tilt mechanism located on the rear end of the vehicle is used for mosaicking and as rear view camera.

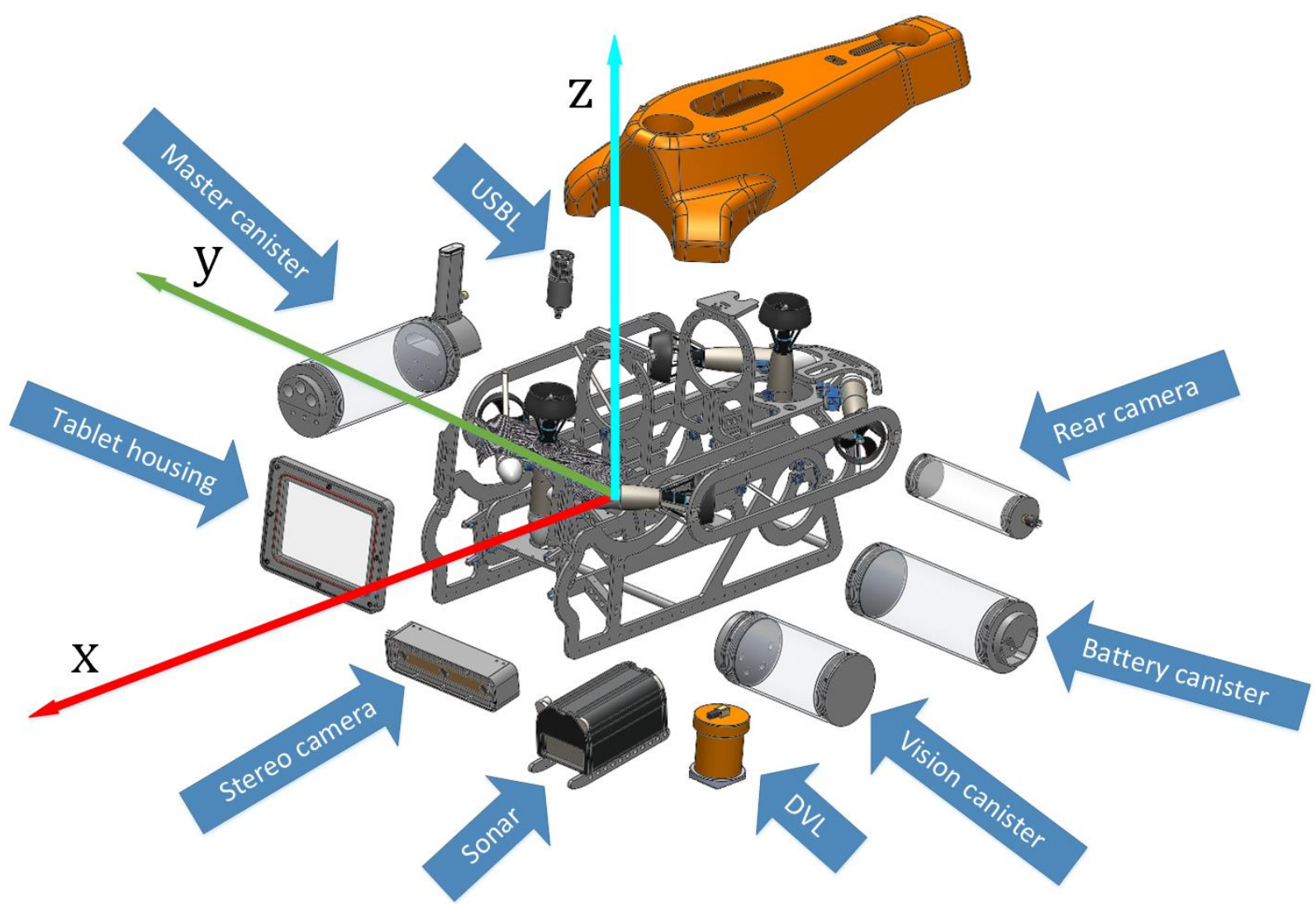

Figure 7 Exploded view of Buddy vehicle 


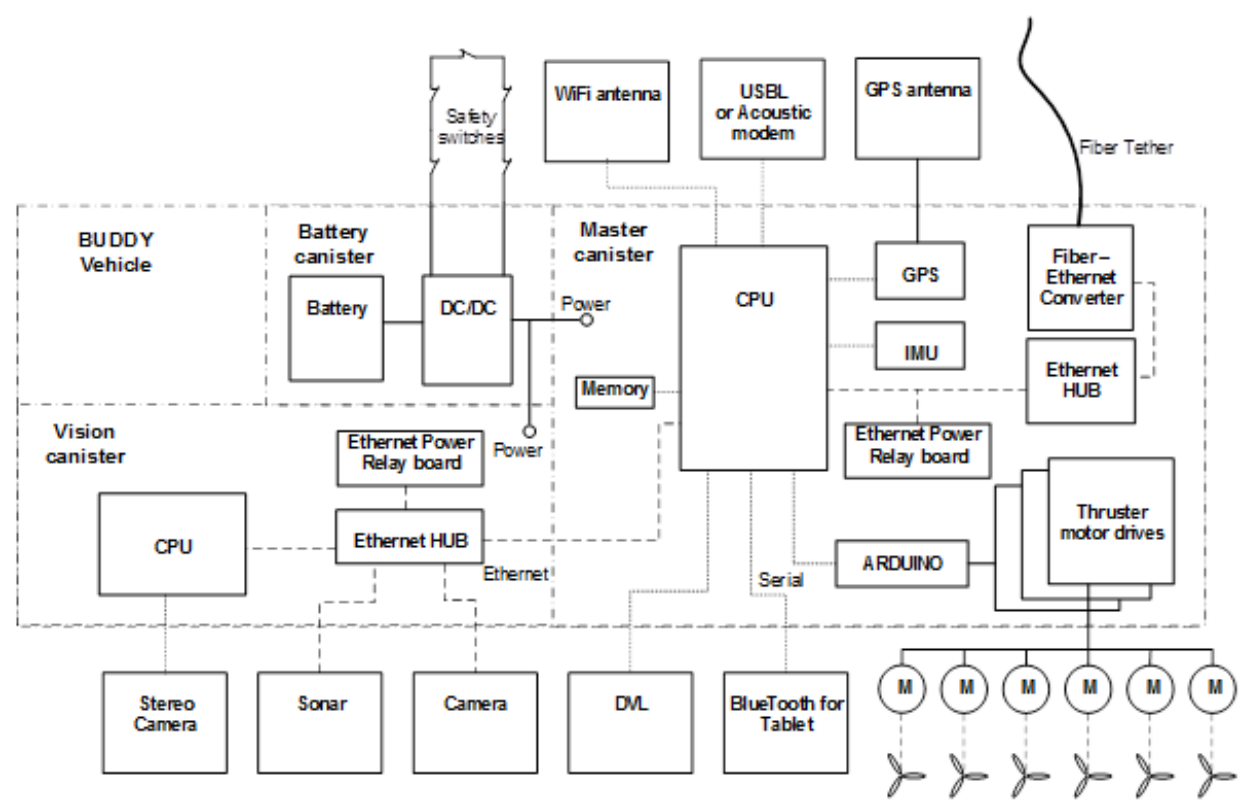

Figure 8 Electrical scheme of the AUV
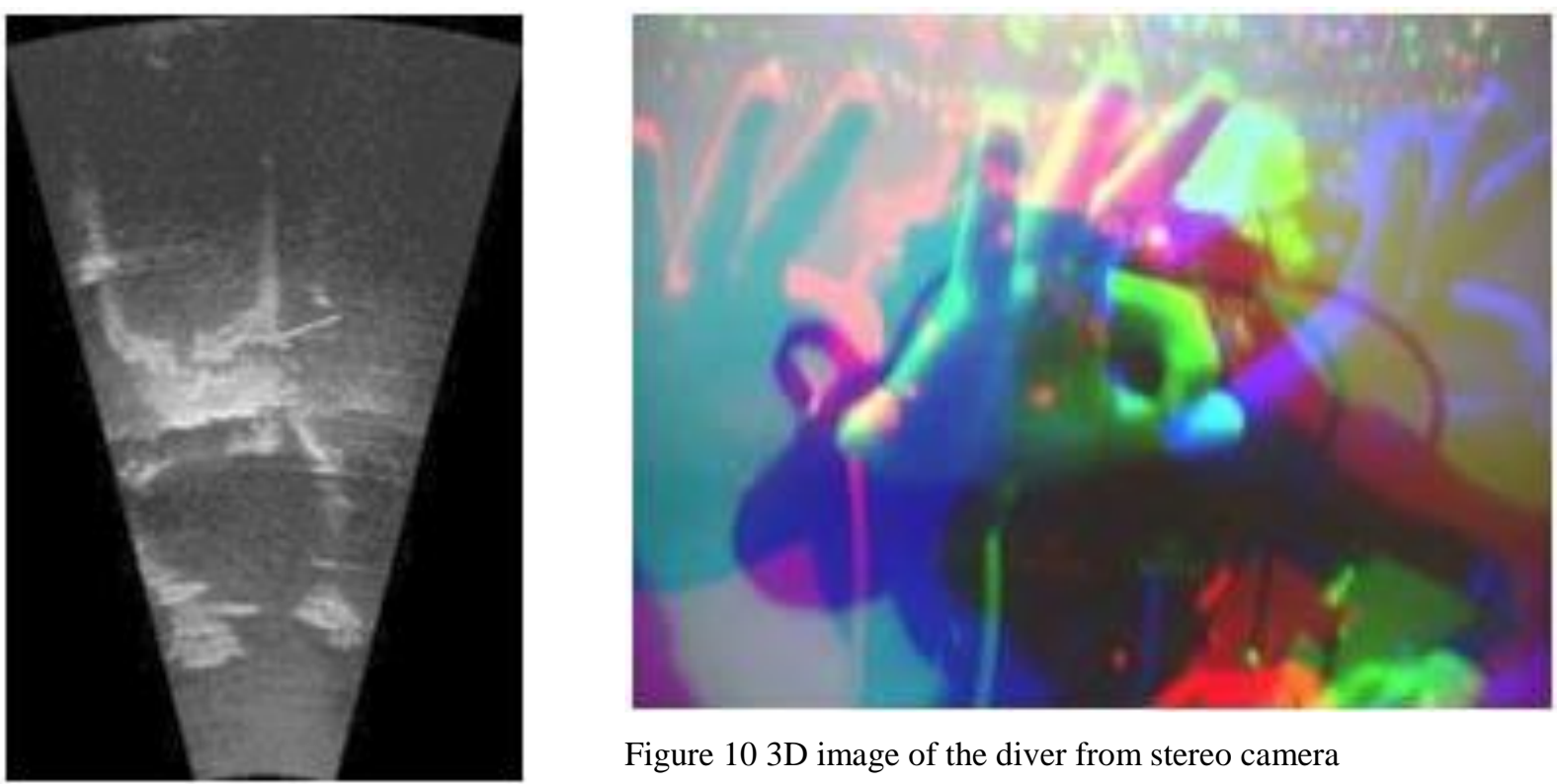

Figure 10 3D image of the diver from stereo camera

Figure 9 Sonar image capturing the diver

\subsection{Thrusters}

The underwater vehicle uses six thrusters for moving in five degrees of freedom (DOF). Four horizontal thrusters set in an X-configuration allow surge, sway and yaw motion. Two vertical thrusters provide control of heave and pitch. Of-the-shelf VideoRay Pro3 thrusters were fitted with a custom made controller. Like on ASV, tests were done to measure thruster forces against the PWM input (Figure 11). Because AUV is not symmetrical like ASV, more complex tests were performed in horizontal plane as well as in vertical plane. Tests included measuring forces in positive and negative thrust for each pair of thrusters:

- Front horizontal thrusters

- Rear horizontal thrusters

- Vertical thrusters. 
Due to fact that thrusters have significantly different forward and backward thrust characteristics and there is a major flow obstruction in the front part of the vehicle (mostly caused by tablet housing), front horizontal and front vertical thruster have reduced efficiency. Thrust measurements have also shown that there are differences between port and starboard thruster pairs in horizontal plane. For that reason, all thrusts forces from thrusters had to be normalized to give decoupled motion. Figure 12 shows that rear starboard thruster gives the biggest thrust of all four thrusters in horizontal plane. As a result all thrusts were normalized to give exactly the same thrust for desired force output given by controller. Analogously, due to flow blocking by multibeam sonar, front vertical thruster has reduced efficiency in vertical plane. Figure 13 shows normalized thrust of the two vertical thrusters illustrating that front vertical thruster gives only half of the thrust of rear vertical thruster. By normalizing thrusts, pitching during descent or ascent is avoided.

\subsection{Underwater tablet housing}

This section provides the description of the dedicated devices for diver-robot interaction. Underwater tablet case was made of Polyacetal (POM-C) and tempered glass. The main purpose of the casing is to protect a commercially available tablet that is used by the diver as an interface to the vehicle. It is situated in the front of the vehicle to ensure easy access for a diver (Figure 7). A customized inductive pen is used for interaction. The tablet case is designed in a symmetrical form to implement $5 \mathrm{~mm}$ thick transparent tempered glass lids onto both sides of the housing. Due to stiffness of tempered glass, less sagging is caused by external pressure. A stainless steel knobs are used for clamping. Rear lid is fastened and independently sealed with the housing frame.

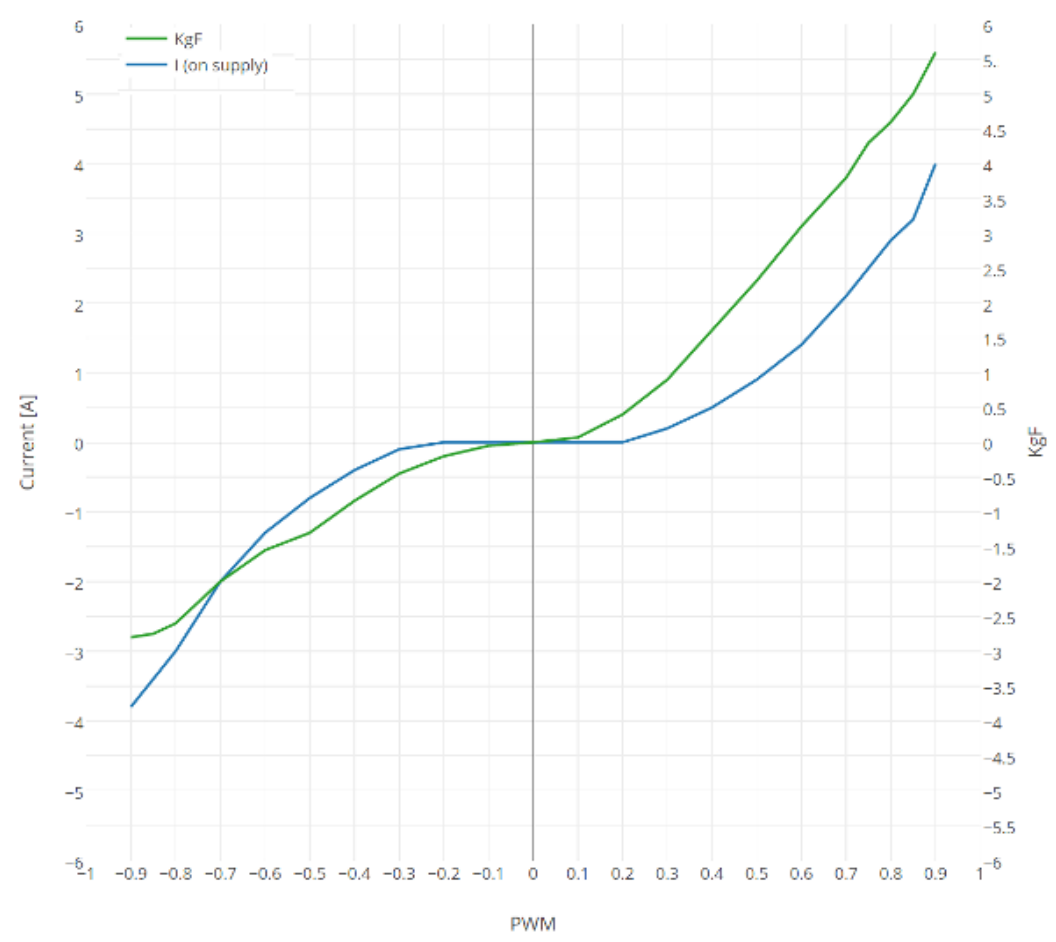

Figure 11 Thrust mapping of Pro3 thrusters 
Nikola Stilinović, Milan Marković

Nikola Mišković, Zoran Vukić, Antonio Vasilijević

\subsection{Diver safety}

In order to ensure diver safety and satisfy obligatory safety requirements, robot has to be equipped with a kill switches, safety mechanism used to shut-off a device in an emergency situation. Activation of the kill switch should completely and as quickly as possible abort the operation. They should be simple to operate and their purpose should be obvious so that even a panicking user with impaired executive function can operate it. A two types of safety kill switches are implemented on the vehicle:

- Magnetic safety switches

- Haptic safety switch.

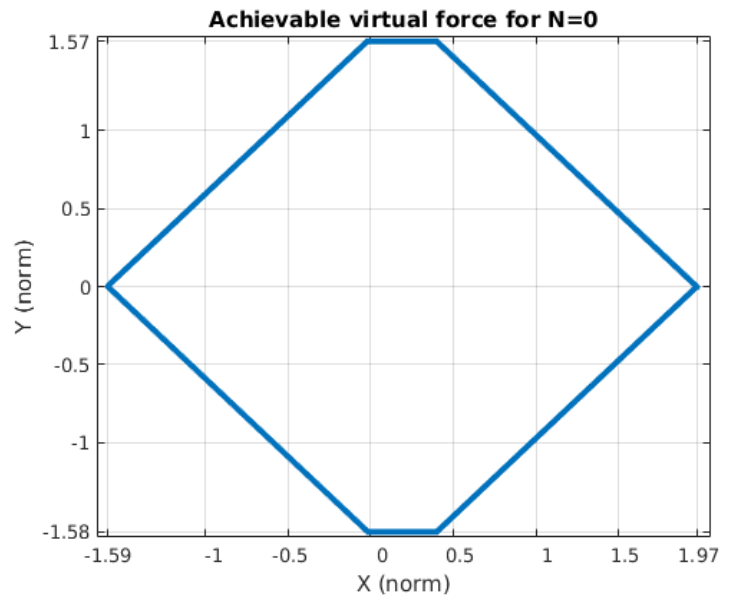

Figure 12 Normalized thrust in motion along $X$ axis in body frame
Mechanical Design of an Autonomous Marine

Robotic System for Interaction with Divers

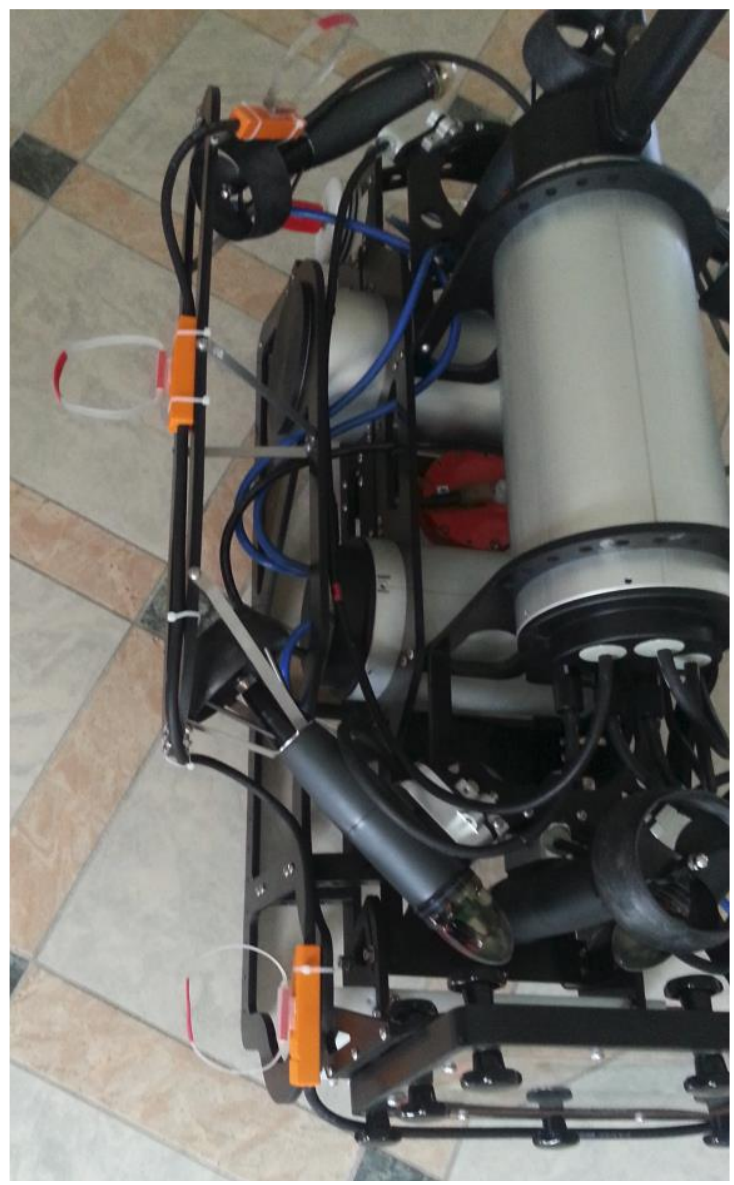

Figure 14 Magnetic kill switches installed on AUV

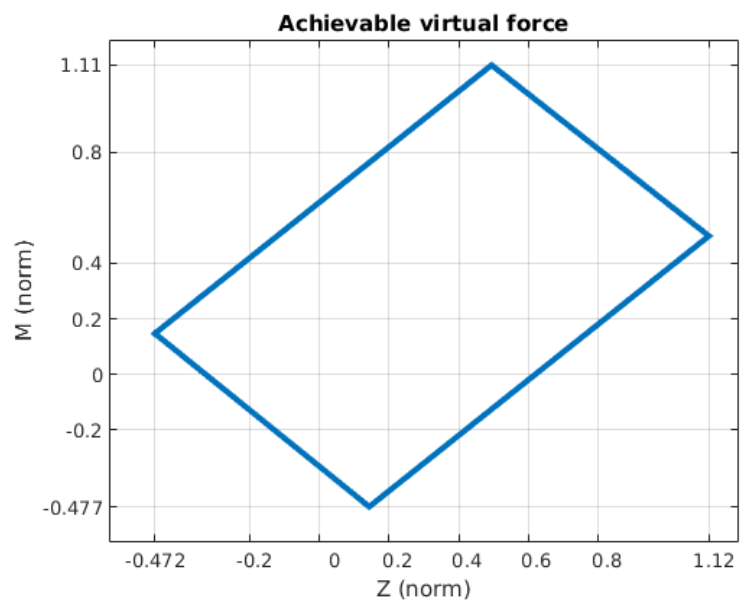

Figure 13 Normalized thrust in motion along $\mathrm{Z}$ axis in body frame

There are five magnetic (reed) relays on the outer shell of the vehicle (Figure 14). Two relays are in front, two on port and starboard sides and one at the back of the vehicle. Magnetic safety switches are directly connected to the solid state relay which powers the thrusters. Relays are connected in series; by removing only one of the five magnets, the solid state relay cuts the power to the thrusters. The haptic safety switch detects changes in IMU 
Mechanical Design of an Autonomous Marine

Robotic System for Interaction with Divers
Nikola Stilinović, Milan Marković

Nikola Mišković, Zoran Vukić, Antonio Vasilijević

readings. If there is any deviation in one of the acceleration axes, e.g. diver pushes the vehicle back if it gets too close, the software automatically stops the thrusters.

\section{Electric assembly}

Buddy vehicle's electronics is divided in three canisters, which interior will be explained in following subsections (Figure 7):

- Battery canister

- Master canister

- Vision canister.

\subsection{Battery canister}

Battery with nominal voltage of $46.8 \mathrm{~V}$ and nominal capacity of $24.8 \mathrm{Ah}(1140 \mathrm{Wh}$ in total $)$ is directly wired to 5,12 and $24 \mathrm{~V}$ DC/DC regulators. Regulators are turned on or off via a magnetic switch which is controlled by a magnet outside the canister. When On/Off pins of DC/DC regulators are short circuited to the ground, regulators are turned off. This is accomplished by putting the magnet over the

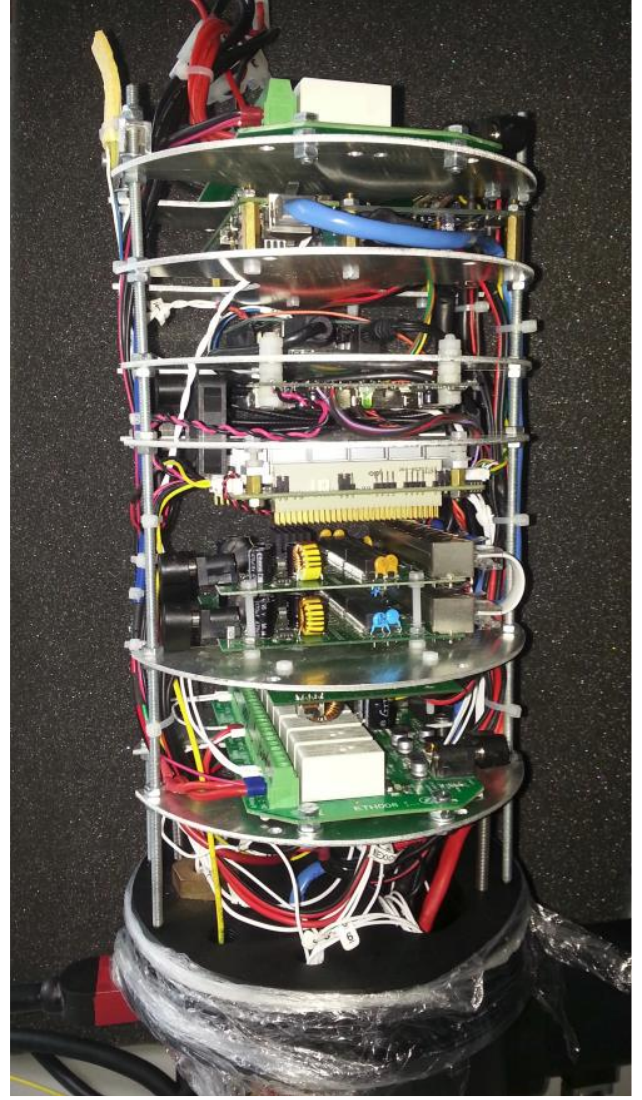

Figure 15 Master cylinder setup magnetic switch. When the magnet is removed, DC/DC regulators are turned on. 24V DC/DC regulator also feeds two $9 \mathrm{~V}$ DC/DC regulators. 5V DC/DC regulator turns on the solid state relay used to power up the motors drivers. The same relay is also controlled by the kill switches. The battery is bonded to one cap of the canister with double sided adhesive tape to prevent movement and rolling inside the canister. Four underwater cables, penetrating the other cap are used to supply Master and Vision canisters. There is an additional magnetic switch, controlled by a magnet outside of the canister, used to completely turn off the battery or reset the battery management system (BMS) in case of e.g. over current or low voltage.

\subsection{Master canister}

As presented in Figure 8 the master canister includes:

- Fiber optic converter for communication with the surface

- Gigabit switch

- Ethernet relay board for powering up thrusters

- Ethernet relay board for powering up CPU, DVL, USBL and GSM module

- PC104 embedded PC

- IMU

- GPS

- Wi-Fi antenna 
- GSM module

- Micro controller

- Motor drives.

Inside the canister is a rack connected to the one of the caps of the canister for easy assembly or disassembly of the whole canister as shown in Figure 15. On the same cap there is antenna shaped bulge protecting Wi-Fi antenna with a GPS module for communication and localization during surface operation and antenna for a GSM module. The GSM module can accept calls or SMS. After receiving a call or SMS the vehicle sends a SMS containing the current telemetry, e.g. GPS position, battery voltage, fault status, etc. Fiber optic cable is connected to a fiber optic converter which is then connected to gigabit Ethernet switch. Overall intention, just as in the ASV, is to have as much equipment as possible connected by Ethernet. This makes equipment easily accessible for the user and allows for independent use of equipment. The equipment not on the Ethernet network is connected to an embedded computer via a serial connection.

The embedded PC is responsible for:

- Acquisition, processing and logging of navigation data from DVL, USBL, IMU, GPS

- Handling of all communication channels: fiber optic, Wi-Fi, GSM and acoustic for sharing the commands and telemetry data with the ground station directly or via surface vehicle

- Running a localisation/navigation filter

- Executing low and high level control algorithms

- Assigning reference to the motor drivers via dedicated microcontroller

- Managing diver-robot interaction using attached tablet.

\subsection{Vision canister}

The Vision canister is responsible for acquisition, processing and logging data from sonar, stereo camera and rear camera. Sonar and rear camera are connected via Ethernet while stereo camera has FireWire link. Sonar and stereo camera gather data of the diver, estimating distance from the AUV. They also recognize diver's pose and sign language. After receiving diver's command, AUV can acknowledge with the message on the underwater tablet. Rear camera can be used for piloting the AUV backwards or it can take the images of sea bottom to make a mosaic. Electronic parts used in Vision canister are:

- Intel NUC Mini PC

- gigabit switch

- Ethernet relay board for powering up CPU, sonar and cameras.

Electronics inside of canister is assembled on rack which is connected to one of the end caps for easy assembly. One of the end caps has 9 pin low profile bulkhead for delivering power. Through other end cap four cables fixed with cable gland and sealed with polyurethane rubber exit the canister:

- 8 wire Ethernet cable for communication with Master canister 
Mechanical Design of an Autonomous Marine

Robotic System for Interaction with Divers
Nikola Stilinović, Milan Marković

Nikola Mišković, Zoran Vukić, Antonio Vasilijević

- 8 wire Ethernet cable for power and communication with low light mono camera and power and communication with tilt control of the camera

- 10 wire Power Ethernet cable for power and communication with multibeam sonar

- 8 wire Ethernet cable acting as FireWire cable for power and communication with stereo camera.

\section{Conclusion}

This paper presents an overview of the mechanical characteristics and functionalities of the marine unmanned vehicles used in CADDY project, supplemented with electrical schemes. Two vehicles are capable of working together in a complex diving support scenario which naturally assumes divers participation.

The main role of the surface vehicle is to be mediator in between air and underwater segments of the CADDY system. The vehicle is equipped with adequate communication systems such as acoustic and $\mathrm{Wi}-\mathrm{Fi}$, to ensure data exchange throughout the fleet and positioning/localisation systems such as GPS and USBL, to ensure spatial situational awareness of the fleet members. To achieve assignments required by the envisioned scenarios, the vehicle control is designed to perform variety of navigation tasks such as dynamic positioning, path following or underwater agent tracking.

The second, Buddy vehicle presented in this paper is unique autonomous vehicle designed for underwater diving support tasks. Integrated underwater table provides possibility for robot-diver interaction adding a new prospective in a diving operations with inclusion of robots. The number of remote sensing systems enable the robot to perform diver observer, guide or slave tasks. The last but not the least is the integrated safety system which is required by and in line with international standards and ensures diver safety when diving in the vicinity of the Buddy vehicle.

The future work involves performing real life experiments with the developed autonomous marine robotic system and human divers. The experiments will mostly be based on testing the human-robot interaction through interpretation of the diver hand symbols. Additionally, focus will be placed on the development of cooperative control algorithms in order to maintain the formation between the diver, and the two autonomous vehicles.

\section{ACKNOWLEDGMENT}

This work is supported by the European Commission under the FP7-ICT project "CADDY - Cognitive Autonomous Diving Buddy" under Grant Agreement No. 611373.

\section{REFERENCES}

[1] http://www.onecaribbean.org/content/files/DivingCaribbeanNicheMarkets.pdf Accessed: 2016-01-18.

[2] Sozer, E.M.; Stojanovic, M.; Proakis, J.G., "Underwater acoustic networks," in Oceanic Engineering, IEEE Journal of , vol.25, no.1, pp.72-83, Jan. 2000 doi: 10.1109/48.820738Reference 3.

[3] N. Fairfield, D. Jonak, G. A. Kantor, and D. Wettergreen, "Field results of the control, navigation, and mapping systems of a hovering AUV," presented at the 15th Int. Symp. Unmanned Untethered Submersible Technol., Durham, NH, Aug. 2007. 
[4] J. Ferrer, A. Elibol, O. Delaunoy, N. Gracias, and R. Garc'1a, "Largearea photo-mosaics using global alignment and navigation data," in Proc. Oceans MTS/IEEE, Vancouver, BC, Canada, Sep./Oct.2007, pp. $1-9$

[5] Ribas, D.; Palomeras, N.; Ridao, P.; Carreras, M.; Mallios, A., "Girona 500 AUV: From Survey to Intervention," in Mechatronics, IEEE/ASME Transactions on , vol.17, no.1, pp.46-53, Feb. 2012 doi: 10.1109/TMECH.2011.2174065

[6] Giacomo Marani, Song K. Choi, Junku Yuh, Underwater autonomous manipulation for intervention missions AUVs, Ocean Engineering, Volume 36, Issue 1, January 2009, Pages 15-23, ISSN 0029-8018, http://dx.doi.org/10.1016/j.oceaneng.2008.08.007.

[7] A. Pedro Aguiar and António M. Pascoal, Cooperative Control of Multiple Autonomous Marine Vehicles: Theoretical Foundations and Practical Issues. In "Recent Advances in Unmanned Marine Vehicles", IET, Editors: Geoff Roberts and Robert Sutton, 2012.

[8] Sattar, J.; Dudek, G., "Where is your dive buddy: tracking humans underwater using spatio-temporal features," in Intelligent Robots and Systems, 2007. IROS 2007. IEEE/RSJ International Conference on , vol., no., pp.3654-3659, Oct. 29 2007-Nov. 2 2007, doi: 10.1109/IROS.2007.4399527

[9] Dudek, G.; Giguere, P.; Prahacs, C.; Saunderson, S.; Sattar, J.; Torres-Mendez, L.-A.; Jenkin, M.; German, A.; Hogue, A.; Ripsman, A.; Zacher, J.; Milios, E.; Hui Liu; Pifu Zhang; Buehler, M.; Georgiades, C., "AQUA: An Amphibious Autonomous Robot," in Computer , vol.40, no.1, pp.46-53, Jan. 2007, doi: 10.1109/MC.2007.6

[10] DeMarco, K.J.; West, M.E.; Howard, A.M., "A simulator for Underwater Human-Robot Interaction scenarios," in Oceans - San Diego, 2013 , vol., no., pp.1-10, 23-27 Sept. 2013

[11] DeMarco, K.J.; West, M.E.; Howard, A.M., "Underwater human-robot communication: A case study with human divers," in Systems, Man and Cybernetics (SMC), 2014 IEEE International Conference on , vol., no., pp.3738-3743, 5-8 Oct. 2014, doi: 10.1109/SMC.2014.6974512

[12] http://www.caddy-fp7.eu Accessed: 2016-01-18.

[13] http://dsor.isr.ist.utl.pt/vehicles/medusa/ Accessed: 2016-01-18.

[14] Mišković, N.; Nađ, Đ.; Stilinović, N.; Vukić, Z.: „Guidance and control of an overactuated autonomous surface platform for diver tracking“", (2013), 21st Mediterranean Conference on Control and Automation, MED 2013 - Conference Proceedings, art. no. 6608884, pp. 1280-1285., DOI:10.1109/MED.2013.6608884re

[15] Vasilijević, A.; Calado, P.; Lopez-Castejon, F.; Hayes, D.; Stilinović, N.; Nađ, Đ.; Mandić, F.; Dias, P.; Gomes, J.; Molina, J.C.; Guerrero, A.; Gilabert, J.; Mišković, N.; Vukić, Z.; Sousa, J.; Georgiou, G.: „Heterogeneous robotic system for underwater oil spill survey,” in OCEANS 2015 - Genova, vol., no., pp.1-7, 18-21 May 2015, DOI: 10.1109/OCEANS-Genova.2015.7271492

[16] Mišković, N., Nađ, Đ., Rendulić, I.: „Tracking Divers: An Autonomous Marine Surface Vehicle to Increase Diver Safety“, (2015), IEEE Robotics and Automation Magazine, 22 (3), art. no. 7258450, pp. 72-84., DOI: 10.1109/MRA.2015.2448851

[17] Vasilijević, A.; Buxton, B.; Sharvit, J.; Stilinović, N.; Nađ, Đ.; Mišković, N.; Planer, D.; Hale, J.; Vukić, Z.: „An ASV for coastal underwater archaeology: The Pladypos survey of Caesarea Maritima, Israel”, (2015) in OCEANS 2015 - Genova, vol., no., pp.1-7, 18-21 May 2015, DOI: 10.1109/OCEANSGenova.2015.7271495

[18] http://www.blueprintsubsea.com/pages/product.php?PN=BP00795 Accessed 2016-06-19

[19] Mišković, N.; Vukić. Z.; Vasilijević, A.: “Autonomous Marine Robots Assisting Divers”, (2013) Lecture Notes in Computer Science - Computer Aided Systems Theory (EUROCAST 2013)

Submitted: $\quad 21.02 .2016 . \quad$ Nikola Stilinović, nikola.stilinovic@ @er.hr Milan Marković

Accepted: $\quad$ 21.07.2016. Nikola Mišković

Zoran Vukić

Antonio Vasilijević

University of Zagreb, Faculty of Electrical Engineering and Computing,

Laboratory for Underwater Systems and Technologies

Unska 3, 10000 Zagreb, Croatia 